\title{
Elevated Liver Enzymes in Asymptomatic Patients - What Should I Do?
}

\author{
Mazyar Malakouti*1 ${ }^{1}$, Archish Kataria*2 ${ }^{2}$ Sayed K. Ali ${ }^{3}$ and Steven Schenker ${ }^{1}$ \\ ${ }^{1}$ Division of Gastroenterology and Nutrition, University of Texas Health Science Center at San Antonio, San Antonio, TX, USA; \\ ${ }^{2}$ Department of Internal Medicine, University of Texas Health Science Center at San Antonio, San Antonio, TX, USA; ${ }^{3}$ Department \\ of Internal Medicine, University of Central Florida, College of Medicine, Orlando, FL, USA
}

\begin{abstract}
Elevated liver enzymes are a common scenario encountered by physicians in clinical practice. For many physicians, however, evaluation of such a problem in patients presenting with no symptoms can be challenging. Evidence supporting a standardized approach to evaluation is lacking. Although alterations of liver enzymes could be a normal physiological phenomenon in certain cases, it may also reflect potential liver injury in others, necessitating its further assessment and management. In this article, we provide a guide to primary care clinicians to interpret abnormal elevation of liver enzymes in asymptomatic patients using a step-wise algorithm. Adopting a schematic approach that classifies enzyme alterations on the basis of pattern (hepatocellular, cholestatic and isolated hyperbilirubinemia), we review an approach to abnormal alteration of liver enzymes within each section, the most common causes of enzyme alteration, and suggest initial investigations.
\end{abstract}

Citation of this article: Malakouti M, Kataria A, Ali SK, Schenker S. Elevated liver enzymes in asymptomatic patients - what should I do? J Clin Transl Hepatol 2017;5(4):394-403. doi: $10.14218 /$ JCTH.2017.00027.

\section{Introduction}

Evaluation of abnormal liver enzyme levels in an otherwise healthy patient can pose a challenge to even an experienced

Keywords: Elevated liver enzymes; Aminotransferase elevation; Liver function tests; Evaluation of abnormal liver enzymes; Approach to alteration of liver enzymes. Abbreviations: $A I H$, autoimmune hepatitis; ALP, alkaline phosphatase; $A L T$, aminotransferase; AMA, antimitochondrial antibodies; anti-HBc, hepatitis B core antibody; anti-HBs, hepatitis $B$ surface antibody; anti-LKM1, anti-liver-kidney microsome-1 antibodies; AST, aspartate aminotransferase; ELISA, enzyme-linked immunosorbent assay; GGT, gamma-glutamyl transferase; GLDH, glutamate dehydrogenase; $\mathrm{HBsAg}$, hepatitis B surface antigen; HBV, hepatitis B virus; $\mathrm{HCV}$, hepatitis $C$ virus; HFE, hemochromatosis gene; IgM anti-HBc, hepatitis B core IgM; IH, ischemic hepatitis; $L D H$, lactate dehydrogenase; MRCP, magnetic resonance cholangiopancreatography; NAFLD, nonalcoholic fatty liver disease; NASH, nonalcoholic steatohepatitis; PBC, primary biliary cholangitis; PSC, primary sclerosing cholangitis; SPEP, serum protein electrophoresis; ULN, upper limit of normal; WD, Wilson's disease.

Received: 24 April 2017; Revised: 22 June 2017; Accepted: 12 July 2017

$*$ Correspondence to: Archish Kataria, Department of Internal Medicine, University of Texas Health Science Center at San Antonio, 7703 Floyd Curl Drive, MC 7878, San Antonio, TX 78229, USA. Tel: +1-210-665-7038, Fax: +1-210-5674856, E-mail: kataria@uthscsa.edu; Mazyar Malakouti, Division of Gastroenterology and Nutrition, University of Texas Health Science Center at San Antonio, 7703 Floyd Curl Drive, MC 7878, San Antonio, TX 78229, USA. Tel: +1-204-803-2523, Fax: +1-210-567-4856, E-mail: malakoutim@uthscsa.edu clinician. It may not be necessary to pursue extensive evaluation for all abnormal test results, however, as it would expose many patients to unnecessary procedural risks and expenses. Conversely, failure to investigate mild or moderate liver enzyme elevations could mean missing the early diagnosis of potential life-threatening yet treatable conditions.

It has become easier and faster to obtain serum liver enzyme levels with automated laboratory testing, which in turn has led to an increase in the number of incidental abnormal findings. It is estimated that about $1 \%$ to $9 \%$ of asymptomatic patients have elevated enzyme levels when screened with standard liver function panels. ${ }^{1,2}$ In a US survey from 1999 to 2002, 8.9\% of the study population showed elevated alanine aminotransferase (ALT) levels, which represents an increase from previous reports. Since no consensus has yet been established, the aim of this review is to provide primary care providers with a systematic approach to interpreting abnormal liver enzymes.

\section{Discussion}

Elevated ALT or aspartate aminotransferase (AST) above the upper limit of normal (ULN; considered to be 30 international units/L for men and 20 for women, with range varying between different labs) in a population without identifiable risk factors should be assessed by physicians, as it is associated with increased liver-related mortality. ${ }^{3}$ However, there are some circumstances in which elevations in liver enzymes are physiological - for example, alkaline phosphatase (ALP) levels may be increased during the third trimester of pregnancy, and both AST and ALT may increase with vigorous exercise. ${ }^{4,5}$

A comprehensive investigation combining thorough history-taking and physical examination, along with diagnostic tests, liver histology and imaging, can often establish a precise diagnosis. The initial approach to an isolated liver enzyme alteration in an apparently healthy person should begin with repeating the test to confirm the result, unless the clinical context points towards an apparent etiology, like a new medication exposure, etc. If the abnormality persists, the evaluation should be based on the magnitude of enzyme elevation. Alteration of liver enzymes can be classified as mild (less $<5 \mathrm{UNL})$, moderate (5-10 UNL) or severe ( $>10 \mathrm{UNL}$ ). This classification is rather subjective, as there is no current consensus of these definitions and various sources use different cut-off points. ${ }^{6-8}$

Once stratification, based on the severity, is completed, the next step is to define the pattern of enzyme elevation, 
which can be divided into three categories, further assisting in clinical evaluation.

- Patterns predominantly reflecting hepatocellular injury ( $\uparrow$ ALT/AST $+/-\uparrow$ bilirubin)

- Patterns predominantly reflecting cholestasis ( $\uparrow$ ALP +/$\uparrow$ bilirubin)

- Mixed ( $\uparrow$ both ALT/AST and ALP)

- Isolated hyperbilirubinemia ( $\uparrow$ bilirubin)

Hepatocellular (Elevated aminotransferases)

\section{Mild hepatocellular pattern of liver enzyme elevation}

Very few prospective studies have addressed a standardized approach to evaluation of the mild hepatocellular pattern of liver enzyme elevation. Some of the landmark studies, conducted by Hultcrantz et al., Friedman et al., Hay et al. and Daniel et al., ${ }^{1,9-11}$ provide insight into the most frequent causes of mild elevation in liver enzymes. These studies also show that the cause of elevated enzyme levels varies greatly, depending on the population studied. Conclusions drawn from the data reported in the literature suggest that fatty liver, resulting either from alcohol use or from nonalcoholic fatty liver disease (NAFLD), is the major cause of mildly elevated aminotransferases and, according to the National Health and Nutritional Survey, point-prevalence of this is about $23 \%$ among American adults. ${ }^{12}$ Some of the drawbacks of the studies include inaccurate reporting of hepatitis $\mathrm{C}$ prevalence (another common cause of liver enzyme alteration) due to unavailability of hepatitis $C$ serologic testing at the time the studies were conducted and lack of a uniform definition of the pathologic diagnosis of nonalcoholic steatohepatitis(NASH). ${ }^{13}$

The first step in the evaluation is to obtain a complete history and perform a thorough physical examination in an effort to identify the most common causes of mildly elevated aminotransferase levels. Some of the important initial questions that will guide further management are: ${ }^{14}$

1. Patient age and ethnicity;

2. Presence of signs and symptoms of chronic liver disease (development of jaundice, edema, pruritus, encephalopathy, gastrointestinal bleeding);

3. Risk factors for viral hepatitis (including but not limited to intravenous/intranasal drug use, body piercings, tattooing, sexual history, travel to foreign countries, occupation);

4. Presence of comorbid conditions like diabetes, obesity, hyperlipidemia for NAFLD, neurologic manifestations in Wilson's disease (WD), emphysema in alpha-1-antitrypsin deficiency;

5. History of alcohol consumption (including history from family), medication use (especially new, careful review of available medical and pharmacy records and laboratory data) and toxin exposure;

6. Family history of genetic conditions pertaining to liver disease, such as hemochromatosis and WD;

7. History of chronic diarrhea or inflammatory bowel disease, indicating extrahepatic causes like celiac sprue, thyroid disorders, inflammatory bowel disease, hereditary and acquired muscle disorders, etc.;
8. Presence of signs and symptoms of heart failure, indicating congestive hepatopathy;

9. History of other autoimmune disorders (i.e. autoimmune hepatitis $(\mathrm{AIH}))$.

Physical examination should be thorough and detailed to look for stigmata of acute and chronic liver diseases which may be subtle or absent, like jaundice (with close attention to the conjunctiva and soft palate), ascites, peripheral edema, hepatosplenomegaly, gynecomastia, testicular hypotrophy, muscle wasting, telangiectasias, palmar erythema, pubic hair changes, etc. ${ }^{14}$ Some liver disorders like hemochromatosis and WD may be associated with specific physical exam findings such as arthritis, acne, skin color changes, KayserFleischer rings, clubbing, etc. ${ }^{14}$ Congestive heart failure would classically present with an elevated jugular venous pressure, hepatomegaly and basilar crackles on auscultation.

If a history of exposure is evident, repeat testing should be undertaken after abstinence from alcohol use, medications and toxins before ordering an extensive work-up. Persistently elevated results on liver function tests, after removal of obvious sources, should be followed by targeted testing based on specific clues from the history and physical exam findings. If the initial assessment from a detailed interview with the patient fails to provide any clues, evaluation should begin with the most common causes of mildly elevated aminotransferase enzymes levels (Table 1). Non-invasive serological tests and imaging procedures may often reveal the most common causes of mild elevations of liver enzymes. If the more common causes have been ruled out and the etiology still remains uncertain, attention should be paid to non-hepatic diseases, such as thyroid disorders, occult celiac disease, etc. ${ }^{15}$

If despite investigation, following a systematic approach (Fig. 1) as outlined above, the cause of mild elevation in aminotransferase levels remains unidentified, then an approach of doing a percutaneous liver biopsy, versus observation alone, may be adopted based on the degree of enzyme elevation. It is acceptable to observe patients, if the levels are less than twice the normal value and no chronic liver condition has been identified through non-invasive tests. This is based on two recent studies which concluded that liver biopsy did not lead to a change in diagnosis or treatment in many such patients and that observation alone proved to be the most cost-effective strategy. ${ }^{16,17}$

Table 1. Common causes of mildly raised aminotransferase levels ${ }^{49}$

- Alcohol
- Medication
- Nonalcoholic fatty liver disease
- Viral hepatitis
- Autoimmune disease
- Congestive heart failure
- Ischemic hepatitis
- Budd-Chiari syndrome
- Alpha-1 antitrypsin deficiency
- Celiac disease
- Endocrine disease: hypothyroidism, Addison's disease
- Disease of striated muscle
- Hemochromatosis
- Wilson's disease
- Glycogen storage diseases




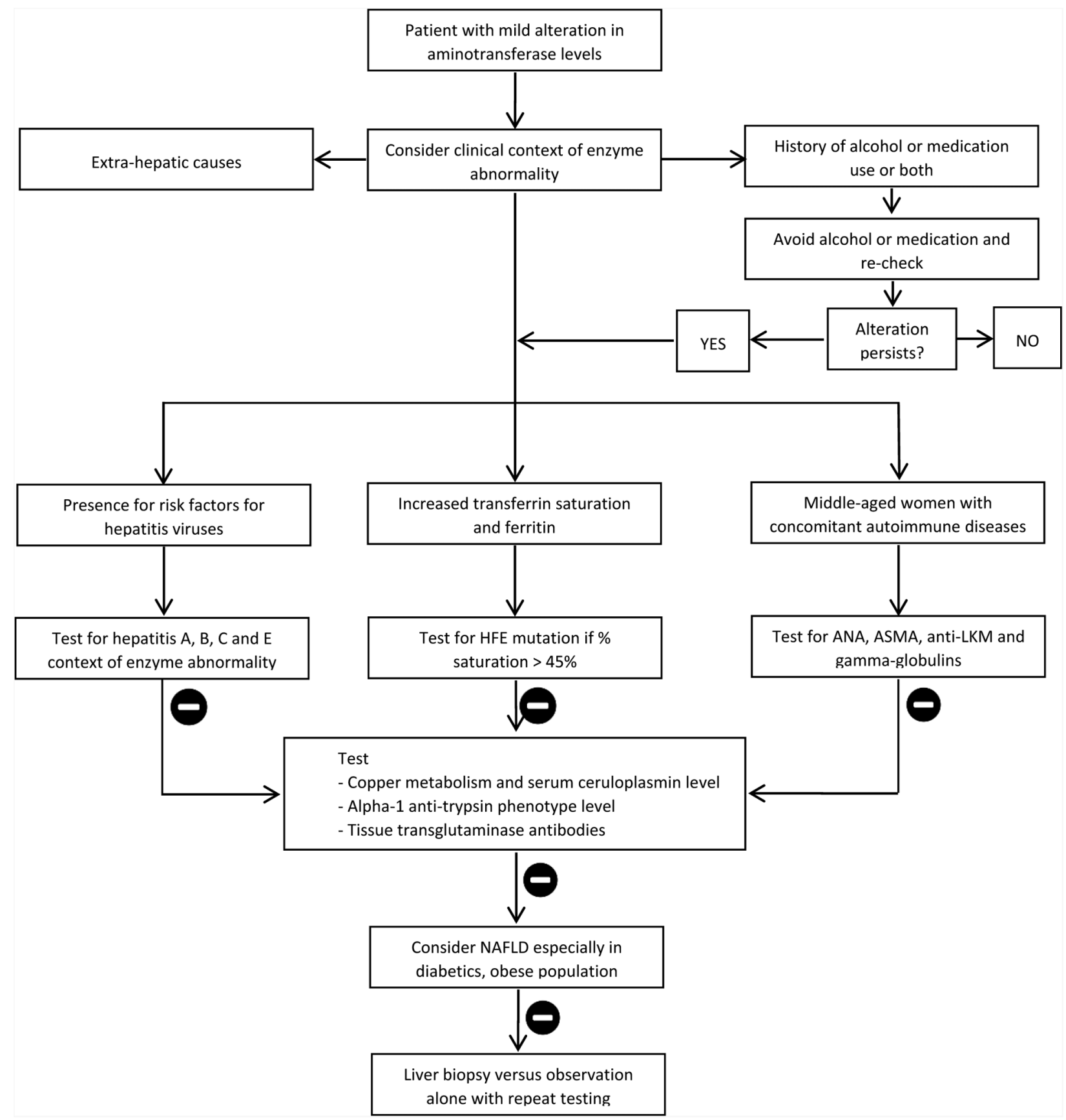

Fig. 1. Schematic initial diagnostic algorithm for a patient presenting with mild aminotransferase abnormality. ${ }^{27}$ Abbreviations: ANA, antinuclear antibody; ASMA, anti-smooth muscle antibody; LKM, anti-liver-kidney microsomal antibody; NAFLD, nonalcoholic fatty liver disease.

Consideration may be given to the effects of decrease in body weight, diabetes control, cessation of alcohol use and other lifestyle modifications. At the same time, it is also important to exclude space occupying lesions and thrombotic disorders of hepatic/portal vasculature like Budd-Chiari syndrome in patients with persistent enzyme elevation and in whom chronic liver disease has been identified. This can be achieved using imaging modalities like ultrasonography and/or computed tomography. A liver biopsy, though unlikely to change management, may be an acceptable approach for providing reassurance to the patient and the physician that no serious disease is present if the levels are persistently more than twice the normal value. ${ }^{17} \mathrm{~A}$ biopsy may also be helpful in assessing the grade of inflammation and when multiple diagnoses are suspected. ${ }^{3}$

\section{Alcohol-related hepatic injury}

An indicator that should make the clinician highly suspicious of alcohol-related liver injury is AST:ALT ratio of 2:1 or more. 
Gamma-glutamyl transferase (GGT) is another sensitive but non-specific marker for hepatic injury which cannot be used solely to diagnose alcohol-related hepatic insult. ${ }^{7}$ Levels of GGT greater than twice the normal values in addition to AST:ALT ratio $>2$ strongly indicate alcohol-induced liver injury as well. ${ }^{18}$

Generally, the enzymes in alcoholic hepatitis are only moderately elevated. The AST seldom exceeds $8-10 \times$ the ULN and the ALT $5 \times$ the ULN; however, in severe acute alcoholic hepatitis the serum bilirubin can rise significantly. ALT levels may even be normal in alcoholic liver disease, and thus a normal serum ALT concentration does not exclude an alcoholic liver disorder. This pattern is thought to be the result of two mechanisms. Most chronic alcoholics are deficient in pyridoxal 5'-phosphate (vitamin B6), which is a necessary coenzyme for both ALT and AST synthesis. Deficiency of pyridoxal 5'-phosphate decreases ALT synthesis to a greater extent than AST synthesis. ${ }^{19}$ Additionally, alcohol itself stimulates the synthesis and release of mitochondrial AST, thereby increasing the AST: ALT ratio. ${ }^{19}$

Although alcohol is a very frequent cause of liver disease, one should not forget to look at comorbid medical conditions like chronic hepatitis B and C, obesity and diabetes mellitus than can also cause liver damage in a patient with alcoholic liver disease. A large, national, population-based study found increased risk of alcohol-related transaminitis with overweight and obesity. ${ }^{20}$

\section{Viral hepatitis}

Incidence of hepatitis $C$ infection is much higher in patients with ALT levels greater than $40 \mathrm{U} / \mathrm{L}$, as compared to the estimated incidence of $1.8 \%$ in the general population. ${ }^{13}$ Incidence of hepatitis $B$ virus (HBV) infection is between $0.2 \%$ and $0.9 \%$ in the general United States' population, which is comparatively less common than hepatitis $\mathrm{C}$ virus (HCV) infection. ${ }^{13}$ However, in patients who have emigrated from endemic areas of the world, the prevalence of HBV infection in the United States can be as high as $20 \% .{ }^{13}$ Risk factors like intravenous drug use, sexual history, travel to foreign countries, occupation, etc. can dramatically increase the prevalence of both viruses. Since these two viruses have such a high prevalence, some clinicians recommend early and empiric testing for HBV and HCV, even in the absence of risk factors for patients presenting with mildly elevated hepatocellular enzymes. ${ }^{21,22}$ Hepatitis $\mathrm{E}$, although uncommon in the United States, should be considered when there is a history of travel to an endemic area (i.e. Central America and Asia).

Most patients with chronic viral hepatitis have minimal elevations in ALT/AST levels, which are generally $<100 \mathrm{U} / \mathrm{L}$. The ratio of ALT:AST is approximately $1 .{ }^{23}$

Initial evaluation for HBV infection includes serologic tests for hepatitis B surface antigen ( $\mathrm{HBsAg}$ ), hepatitis B surface antibody (anti-HBs) and hepatitis B core antibody (anti-HBc). Interpretations of these serological markers are discussed in Table 2. Serologic tests that indicate presence of viral replication and infectivity include hepatitis $B$ e antigen, hepatitis $B$ e antibody, and HBV DNA. Occult HBV infection is characterized by the presence of HBV DNA in the absence of detectable HBs antigen. ${ }^{24}$ Some occult HBV infections may manifest with negative hepatitis $B$ e antigen but positive HBV DNA polymerase. Biopsy should be considered in patients that are positive for HBV DNA and hepatitis B e antigen, to assess the severity of disease. ${ }^{7}$

The initial screening test for HCV infection begins with serologic test for HCV antibody which is very sensitive, but false positive tests are frequent even with third generation enzyme-linked immunosorbent assays (ELISAs) as seen in hypergammaglobulinemia of AIH. Addition of the recombinant immunoblot assay for anti-hepatitis $C$ can improve the specificity of ELISA testing. HCV infection can be confirmed with a PCR assay that detects serum HCV RNA (reflecting active viral infection and replication). ${ }^{25}$ PCR testing can be performed by quantitative or qualitative methods. If negative, an HCV RNA PCR test should be repeated in 3 months in individuals who screen positive for HCV infection, to ensure that it was not a false negative. ${ }^{25}$ Quantitative single real-time PCR assays can also be used to assess virologic response to treatment as part of therapeutic management of hepatitis C. ${ }^{26}$

Table 2. Interpretation and clinical significance of hepatitis B serologies

\begin{tabular}{|c|c|c|c|c|}
\hline $\mathrm{HBsAg}$ & Anti-HBc & Anti-HBs & IgM anti-HBc & Interpretation \\
\hline Negative & Negative & Negative & & Susceptible \\
\hline Negative & Negative & $\begin{array}{l}\text { Positive, } \\
>10 \mathrm{mIU} / \mathrm{mL}\end{array}$ & & Immune due to vaccination \\
\hline Negative & Positive & Positive & & Immune due to natural infection \\
\hline Positive & Negative & Negative & Negative & Early acute infection \\
\hline Positive & Positive & Negative & Positive & Acutely infection \\
\hline Positive & Positive & Negative & Negative & Chronic infection \\
\hline Negative & Positive & Negative & & $\begin{array}{l}\text { Either: } \\
\text { 1. Recovering from acute HBV infection } \\
\text { 2. Distantly immune: test not sensitive enough to detect a very low } \\
\text { level of anti-HBs in serum } \\
\text { 3. Susceptible with a false positive anti-HBC } \\
\text { 4. Chronically infected with undetectable level of HBsAg present in } \\
\text { serum }\end{array}$ \\
\hline
\end{tabular}


In patients who test positive for HBV DNA and HCV RNA, liver biopsy is recommended to assess the stage of fibrosis, evaluate the need for therapy, establish a prognosis and assess progression. ${ }^{27}$

Degree of aminotransferase alteration together with an AST:ALT ratio $>1^{28-31}$ in patients with chronic viral hepatitis seems to prognosticate poor outcomes with more likelihood of progression to cirrhosis. ${ }^{20}$ This observation is supported by the fact that AST:ALT ratio $>1$ can be found in about $79 \%$ of patients with cirrhosis secondary to viral etiology, in contrast to only $4 \%$ of patients with chronic viral hepatitis alone. ${ }^{29}$ Such patients were shown to have 1 -year survival, ${ }^{28}$ with $87 \%$ sensitivity $(69 \%-96 \%, 95 \% \mathrm{CI})$ and $52 \%$ specificity $(40 \%-64 \%, 95 \% \mathrm{CI}) .^{31}$ It may be beneficial to obtain an ultrasound image for such patients, to look for the presence of liver masses. ${ }^{27}$

\section{NAFLD}

NAFLD is an entity that includes a spectrum of diseases ranging from simple steatosis to $\mathrm{NASH}$ to cirrhosis. As previously mentioned it is the most common cause of mild aminotransferase alteration in the general population of the United States. It rarely causes severe or fulminant rise in enzymes that lead to liver failure. Prevalence of hepatic steatosis is reported to be about $25 \%$ in the general United States' population, which is much higher than that of its progressive form (NASH, 3-5\%). ${ }^{13}$ Certain high risk patient groups, like type 2 diabetics and morbidly obese patients undergoing bariatric surgery, have a much higher prevalence of NAFLD compared to the general population.

NAFLD is a diagnosis of exclusion. The only laboratory evidence of NAFLD may be mild elevations in liver enzymes. ALT is usually greater that AST, making the AST:ALT ratio $<1 .^{32,33} \mathrm{GGT}$ levels can be elevated up to 3 times the upper reference value in about $50 \%$ of patients with NAFLD, even in the absence of alcohol consumption. ${ }^{33-35}$ Reversal of the AST:ALT ratio is a marker for poor prognosis, suggesting initial or advanced fibrosis ${ }^{36}$ that can be explained due to increased mitochondrial damage and decreased hepatic clearance of AST with advancing liver disease. ${ }^{20}$

The synthetic function of liver as measured by total bilirubin and albumin is usually preserved in early NAFLD without cirrhosis. Additionally, leucopenia and thrombocytopenia should make the clinician suspicious for the presence of cirrhosis and occult portal hypertension. Imaging modalities like ultrasonography or computed tomography can help identify fatty infiltration of the liver, but the gold standard for confirming NAFLD remains liver biopsy. ${ }^{12,37,38}$ Histology shows fatty infiltration with peri-central fibrosis, inflammation and necrosis of hepatocytes. In addition, hyaline cytoplasmic inclusions may be found in hepatocytes that appear identical to Mallory bodies, which are characteristic of alcoholic liver disease. ${ }^{35}$ Treatment is mainly directed towards weight loss and addressing the underlying factors or comorbidities. ${ }^{39}$ Alternative treatment may include supplementation of vitamin E. However, it is still being studied and is not used widely; in two pilot studies ${ }^{40}$ it was shown to decrease transaminase levels and reverse histologic abnormalities.

\section{Hemochromatosis}

Hereditary hemochromatosis should be considered early in the evaluation of men with elevated liver enzymes, especially those of northern European descent as the prevalence of this entity is $0.25 \%$ to $0.5 \%$ in such people. Clinically, patients remain asymptomatic until iron overload causes significant end-organ damage.

Screening for hereditary hemochromatosis can be done by measuring serum iron levels and total iron-binding capacity. A positive screening test is defined as individuals with a transferrin-saturation value (ratio of serum iron level to the total iron-binding capacity) $>45 \% .{ }^{41}$ Being an acute-phase reactant, serum ferritin can be falsely elevated in acute inflammatory conditions and therefore its measurement provides less specific information. Individuals with a positive screening test should undergo liver biopsy to measure hepatic iron levels and assess the severity of liver damage. A hepatic iron index can then be calculated according to the ratio of hepatic iron level in micromoles per gram of dry weight to the patient's age. Levels $>1.9$ predict presence of homozygous hereditary hemochromatosis. ${ }^{41}$

Genetic testing, which has decreased the need for liver biopsy, has now become available to identify the mutation in the hemochromatosis (HFE) gene that causes the majority of cases. Being non-invasive, it seems like an attractive option; yet, genetic testing has failed to replace liver biopsy as the gold standard for confirmatory diagnosis due to its lack of sensitivity. ${ }^{42}$ Two key point mutations (C282Y and H63D) linked to hereditary hemochromatosis have been identified. Individuals who are homozygous for the $\mathrm{C} 282 \mathrm{Y}$ mutation seem to carry the greatest risk of iron overload. The compound heterozygote (C282Y/H63D) and occasionally the H63D homozygote patient populations can also present with iron overload in a minority of cases. Liver biopsy in hereditary hemochromatosis patients who are younger than 40 years of age and who have normal liver tests is usually not necessary. ${ }^{7}$

In patients with hemochromatosis, due to the increased risk of hepatocellular carcinoma, it is important to exclude cirrhosis. Such patients may benefit from bi-annual ultrasound and $\alpha$-fetoprotein level measurement for screening purposes. Presence of certain markers like ferritin $<1000 \mu \mathrm{g} / \mathrm{L}$, normal AST values, and absence of hepatomegaly were shown to accurately exclude cirrhosis in C282Y homozygotes in a recent study. ${ }^{43}$ However, a serum ferritin level of $>1000$ $\mu \mathrm{g} / \mathrm{L}$ has a poor positive predictive value, and so a liver biopsy in such patients may aid in diagnosing cirrhosis due to hemochromatosis. Similar information is not available for nonC282Y homozygotes.

\section{WD}

WD may present only with elevation of liver enzymes and no other clinical symptoms. It has a homozygote frequency of $1: 30000-1: 300000 .{ }^{27}$ Onset of clinical symptoms occur between the ages of 5-25 years, although some patients may present up to the 4th decade of life. Initial evaluation or screening includes testing for serum ceruloplasmin levels (reduced in $85 \%$ of patients with WD). ${ }^{7}$ Slit-lamp examination for Kayser-Fleischer rings is a useful clinical clue. The 24-hr urinary copper excretion should be checked ( $>100 \mu \mathrm{g} /$ day of copper is suggestive) in patients suspected of WD who have normal ceruloplasmin and absent Kayser-Fleischer rings. A copper concentration of more than $250 \mu \mathrm{g} / \mathrm{g}$ dry liver weight on liver biopsy confirms the diagnosis. As there are numerous genotypic patterns associated with this disease, it is not useful to make the diagnosis of WD with molecular or genetic testing. ${ }^{7}$ 


\section{Alpha-1 antitrypsin deficiency}

The disease incidence in Europe and the United States is $1: 1600-1: 2800$. It is an uncommon cause of chronic liver disease in adults and it is usually identified in early childhood, with a very small percentage progressing to cirrhosis in adulthood. ${ }^{44}$ Screening test in suspected patients with concomitant pulmonary disease (emphysema) involves detecting low levels of alpha1-antitrypsin in the serum or by the lack of a rise in $\alpha$-globulin bands on serum protein electrophoresis (SPEP). ${ }^{7}$ However, as alpha 1 -antitrypsin levels may be increased in inflammatory conditions, thereby causing a false negative result; a phenotype determination is usually necessary to confirm the diagnosis. ${ }^{7}$

\section{AIH}

AIH primarily occurs in young to middle-aged women with concomitant autoimmune disorders (e.g., autoimmune thyroiditis, connective tissue diseases) and often showing a female to male predominance (ratio of $4: 1$ ). ${ }^{45,46}$ The disease prevalence is about $1: 6000$ to $1: 7000$, and as many as $80 \%$ of patients may have hypergammaglobulinemia on SPEP, which is useful for screening purposes even in the absence of cirrhosis. ${ }^{45,47}$ Polyclonal immunoglobulins more than twice the normal level is most suggestive of the diagnosis, however additional tests such as measurement of antinuclear antibodies, antibodies against smooth muscle and liver-kidney microsomal antibodies (anti-LKM1), support the diagnosis and also help typify the disease. Liver biopsy can be performed to confirm the diagnosis, if all other tests are inconclusive. $45,47,48$

Type $1 \mathrm{AIH}$ is the most common form of autoimmune disease worldwide. Predominantly a disease of women less than 40 years of age, type $1 \mathrm{AIH}$ is characterized by the presence of antinuclear antibody and/or smooth muscle (actin) antibodies. ${ }^{49}$ Type $2 \mathrm{AIH}$ is predominantly a disease of children aged 2-14 years ${ }^{49}$ and is characterized by the presence of anti-LKM1. It is commonly associated with other autoimmune disorders like type 1 diabetes, vitiligo, autoimmune thyroiditis and pernicious anemia. Low serum immunoglobulin A levels may be present in such patients. ${ }^{49}$ Type $3 \mathrm{AIH}$ is characterized by the presence of antibodies to soluble liver/liver-pancreas antigen; however, this disease form is not well recognized. ${ }^{49}$

Initiation of corticosteroids results in significant therapeutic response (supports diagnosis), but just like any other chronic autoimmune disease, patients may have intermittent flares which mimic acute hepatitis. ${ }^{45,47,48}$

\section{Drug-related liver injury}

Specific query for common drug classes (especially acetaminophen), non-prescribed herbal supplements and illicit drugs (illustrated in Table 3 ) is essential to discovery of associated liver injury. It is difficult to attribute liver injury to a specific drug when patients with several co-morbidities are on multiple medications. In such cases, it may be necessary to empirically discontinue a suspected medication or replace with an alternative and monitor for recovery of liver chemistries. Liver biopsy helps in determining the severity of liver injury caused by the offending agent and is indicated in cases of acute fulminant liver failure. ${ }^{3}$
Table 3. Medications, illicit drugs and herbs reported to cause elevation in liver enzyme levels ${ }^{49}$

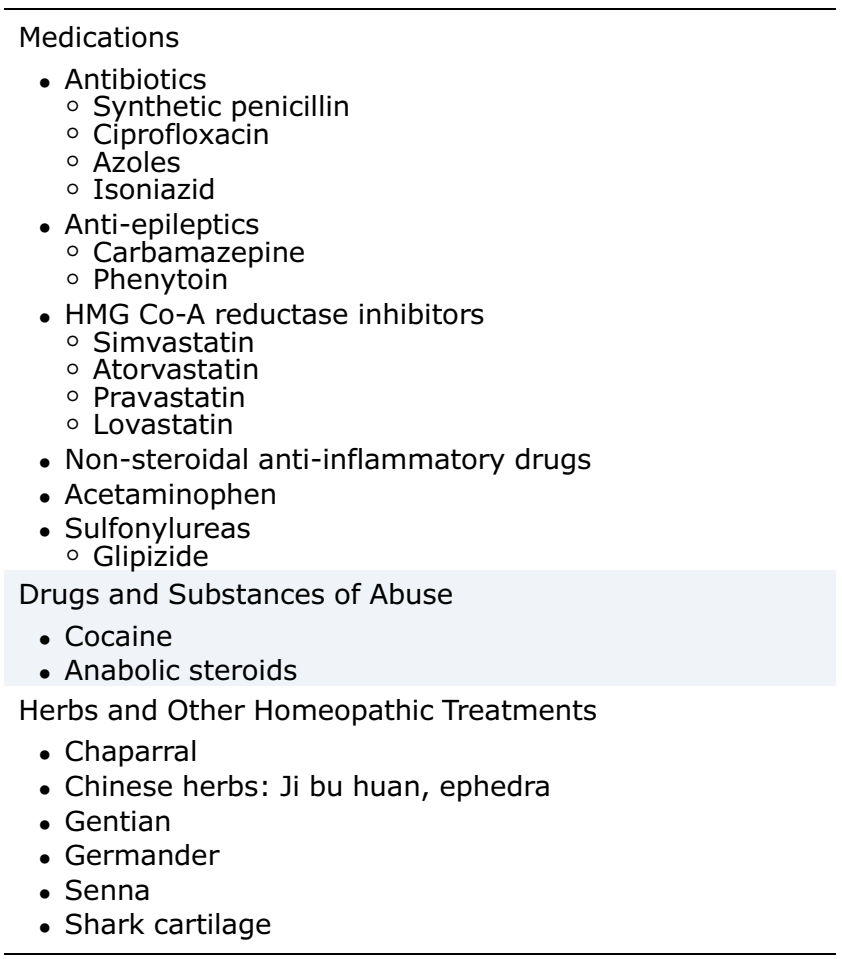

\section{Non-hepatic causes (summarized in Table 4)}

Celiac disease may be found in approximately $5-10 \%$ of patients with unexplained elevation of aminotransferase levels, even without gastrointestinal symptoms. ${ }^{50-52}$ This finding is based on a recent study where celiac sprue was a cause of asymptomatic aminotransferase elevation in 13 of 140 patients referred to the liver clinic. ${ }^{50}$ The presumptive diagnosis can be made by the presence of decreased serum levels of tissue transglutaminase-IgA antibodies (assuming total IgA antibody level is normal). Upper endoscopy-guided small bowel biopsy may be pursued afterwards to confirm the diagnosis and to grade the disease. ${ }^{53}$ Most patients with celiac disease and altered aminotransferase levels are found to have mild steatosis and minimal inflammatory changes on liver histopathology, with no relation to aminotransferase levels or degree of steatosis. ${ }^{23}$

Aminotransferase elevation, especially that of AST, is very non-specific as it is also abundantly present in other tissues, such as striated muscles, red blood cells, etc. Apart from liver disease, elevations in AST levels may also result from inborn errors of muscle metabolism, acquired muscle disorders such as myositis and rhabdomyolysis (from strenuous exercise), and hemolysis. ${ }^{7}$ Screening for such conditions can be done by serum creatinine kinase or aldolase levels. ${ }^{7}$

Although granulomatous disorders like tuberculosis, sarcoidosis, amyloidosis and metastatic or primary hepatocellular carcinoma usually have ALP level alterations (up to 20-fold rise in ALP depending upon the extent of involvement), they can also have mild to moderate elevations of aminotransferase levels. 
Table 4. Extrahepatic causes of elevated serum aminotransferase levels ${ }^{23}$

Myocardial infarction

Muscle disease

- Hereditary (dystrophies, metabolic abnormalities)

- Acquired (myositis, traumatic rhabdomyolysis ${ }^{*}$, cramps) Hyper- and hypothyroidism

Addison's disease

Celiac disease

Inflammatory bowel disease

Congestive heart failure

Heat stroke

Malignant hyperthermia

Strenuous physical activity

*In acute rhabdomyolysis, initially the AST-ALT ratio is $>3$, but secondary to shorter half-life and faster decrease of AST levels, the ratio approaches 1 after a few days. Therefore, patients with chronic muscle disease have approximately equal serum AST and ALT concentrations.

Moderate/severe hepatocellular pattern of liver enzyme elevation

This degree of enzyme elevation can be associated with (but not limited to) ischemic/toxic damage to the liver, acute viral hepatitis, medications, acute biliary obstruction and alcohol abuse. ${ }^{27}$ This condition should be evaluated expeditiously, since the rate of change in the degree of enzyme elevation with respect to time is acute, especially if symptoms of hepatic decompensation are present.

As with the mild pattern of liver enzyme elevation, investigation should begin with a thorough history-taking and physical examination. Detailed review of the patient's pharmacologic chart is crucial to identifying hepatotoxic medications and herbal products. ${ }^{54-56}$ Risk factors for hepatitis can be assessed in a fashion similar to that done for mild aminotransferase elevation, which may help identify the cause and drive the subsequent investigation. History of exposure to risk factors may be lacking in some patients with acute viral hepatitis. Clinical symptoms such as fatigue, arthralgia, lowgrade fever or jaundice are more common in patients with acute hepatitis A $(70-80 \%)$ or B (30-50\%), as compared to patients with acute hepatitis C $(20 \%) \cdot{ }^{4,57}$ Complaints of abdominal pain, fever and jaundice (Charcot's triad) would point towards a diagnosis of acute biliary obstruction. Presence of shock (septic, cardiogenic, hemorrhagic, etc.) from varied etiologies may indicate ischemic hepatitis $(\mathrm{IH}) .^{58-60}$

Initial history-taking and physical examination should be followed up with evaluating the magnitude and rate of change of aminotransferase alteration with respect to time course of injury, as it may provide a clue towards the differential diagnosis. ${ }^{27}$ Ischemic or toxic damage to the liver usually causes extremely high aminotransferase levels in about $90 \%$ of the cases ( $>75$ times the ULN) in contrast to viral hepatic injury, in which such high levels are usually not present. Biochemical features of common causes of moderate to marked increase in aminotransferase levels are outlined in Table 5.

\section{IH}

IH typically presents with acute rise and rapid decrease of aminotransferase levels, including lactate dehydrogenase $(\mathrm{LDH})$, to normal values within a few days. Levels of enzymes are extremely high, with peak values quite often reaching $>5000 \mathrm{U} / \mathrm{L}$. The ALT/LDH ratio is typically $<1$. AST levels usually peak before those of ALT because of the enzyme's peculiar intra-lobular distribution. ${ }^{59-61}$ Rise in glutamate dehydrogenase (GLDH) signifies ischemic insult to the liver as the enzyme is localized in the center of the lobule (zone 3 of the acinus), which is most prone to hypoxic injury. In about $80 \%$ of patients with ischemic injury, the serum bilirubin level is lower than $34 \mathrm{mg} / \mathrm{dL}$.

It is essential to note that a decline in aminotransferase levels alone does not necessarily signify improvement in the patient's condition, since both resolution and massive hepatic necrosis may demonstrate a similar biochemical picture. Measurement of serum bilirubin and prothrombin time helps differentiate the two scenarios and should be closely monitored in the latter case for the potential risk of hepatic failure. There are no specific serologic tests to diagnose ischemic liver injury. A low threshold for diagnosis should be maintained in low-flow hemodynamic states, and prognosis depends on the underlying illness. ${ }^{27}$

Table 5. Biochemical features of common causes of moderate to marked increase in aminotransferase levels ${ }^{27}$

\begin{tabular}{|c|c|c|c|}
\hline Cause & $\begin{array}{l}\text { Aminotransferase } \\
\text { level increase } \\
\text { (value } \times \text { URL) }\end{array}$ & $\begin{array}{l}\text { Bilirubin level } \\
\text { increase } \\
\text { (value } \times \text { URL) }\end{array}$ & Comments \\
\hline Ischemic injury & $>10$ to $>50$ & $<5$ & $\begin{array}{l}\text { AST > ALT; rapid rise and fall of aminotransferase levels; } \\
\text { ALT/LDH ratio }<1 \text {; presence of comorbid conditions }\end{array}$ \\
\hline Toxic injury & $>10$ & $<5$ & $\begin{array}{l}\text { AST > ALT; rapid rise and fall of aminotransferase levels; } \\
\text { history indicative of toxic injury }\end{array}$ \\
\hline Acute viral hepatitis & $5-10$ to $>10$ & $5-10$ & $\begin{array}{l}\text { Slow decrease of aminotransferase levels; presence of risk } \\
\text { factors }\end{array}$ \\
\hline $\begin{array}{l}\text { Acute biliary } \\
\text { obstruction }\end{array}$ & $5-10$ & $5-10$ to $>10$ & $\begin{array}{l}\text { Aminotransferase increase may precede cholestasis; } \\
\text { Charcot's triad }\end{array}$ \\
\hline Alcoholic hepatitis & $5-10$ & $5-10$ to $>10$ & $\begin{array}{l}\text { AST/ALT ratio }>2 \text {; may occur as both acute and } \\
\text { acute-on-chronic injury }\end{array}$ \\
\hline
\end{tabular}




\section{Toxins/medications}

The pattern of enzyme alteration is similar to that of IH. Laboratory testing is only reserved for cases of acetaminophen poisoning for which drug serum levels can be measured. Interpretation of a toxic level depends on the time since ingestion of acetaminophen using the Rumack-Matthew nomogram, which serves as a helpful guide to therapy. ${ }^{62}$

\section{Acute viral hepatitis}

ALT levels are higher than AST levels in uncomplicated acute hepatitis $A$ and $B$, with both levels being well above $1,000 \mathrm{U} / \mathrm{L}$, contrary to hepatitis $C$ where there is a moderate elevation in liver enzymes. ${ }^{63}$ Decline of aminotransferase levels typically occurs slowly and gradually over several weeks. A rapid, precipitous fall within several days is prognostically unfavorable, indicating "exhaustion" of the liver. It is suggested to monitor the hepatic synthetic function (e.g., prothrombin time) in such situations, as there is risk for potential liver failure. ${ }^{23}$ Enzyme levels usually peak before jaundice appears, and there is a greater increase in serum bilirubin levels compared to ischemic/toxic injury. About $70 \%$ of cases of acute hepatitis $A, 33-50 \%$ of cases of acute hepatitis B and $20-33 \%$ of cases of acute hepatitis $C$ present with jaundice as one of the clinical signs. ${ }^{4}$

Hepatitis A IgM and hepatitis B core IgM (IgM anti-HBC) antibodies, $\mathrm{HBsAg}$ and $\mathrm{HCV}$ antibodies can be tested as part of the laboratory evaluation for acute viral hepatitis. If HCV antibodies are positive, it may be beneficial to test further for HCV RNA, in order to differentiate between a past and an acute infection. Although these patients are at very low risk of developing acute hepatic failure, recent data shows that they may benefit from early therapeutic intervention to prevent chronic complications. ${ }^{64}$ Hepatitis D can only occur in patients that are positive for $\mathrm{HBsAg}$, as it requires viral particles of HBV for its own replication and infection. Therefore, testing for hepatitis $D$ should only be done in patients that are positive for HBsAg. Additionally, in the case of moderate to severe elevation of aminotransferase levels, testing for acute hepatitis $E$ (IgM of hepatitis E virus) should also be considered in those returning from endemic areas and whose tests for acute hepatitis $A, B$ and $C$ are negative.

Once the most frequent causes of moderate to severe aminotransferase alteration have been ruled out, the clinician should broaden the differential diagnosis to include minor hepatitis viruses (e.g., hepatitis E, Epstein-Barr virus, cytomegalovirus, etc.), WD, hemochromatosis, and autoimmune, extrahepatic and congenital causes. ${ }^{6-8,65}$ Up to $49 \%$ of patients with AIH present with moderate increase in aminotransferase levels and jaundice. ${ }^{45,48,57}$ Patients with acute extrahepatic biliary obstruction can have very high AST levels (up to 10 times the ULN, with peak $>50$ times the ULN in $1-2 \%$ of patients) that rapidly decrease once the biliary tree is decompressed. ${ }^{4,66-68}$ Findings of dilated bile ducts on ultrasound/magnetic resonance cholangiopancreatography (MRCP) usually provide definitive diagnosis.

If there are signs of acute liver failure, urgent hepatology consultation with referral to a liver transplant center should be undertaken immediately. Finally, if all diagnostic evaluation is negative for moderate to severe aminotransferase elevation, a liver biopsy should be considered if the patient is medically stable.

\section{Cholestasis}

\section{Elevated ALP}

ALP is an enzyme that is responsible for transportation of metabolites across the cell membrane. Liver and bone diseases are the most common causes of pathological ALP level elevation. In some patients, the cause of ALP elevation could be physiological (i.e. pregnant women, adolescents). The degree and rate of elevation may provide only minor clues to the diagnoses however, and the patient's history and physical exam findings may help significantly in reaching the diagnosis. Obtaining GGT levels and a liver ultrasound may provide valuable results.

Ultrasound or other imaging modalities can exclude biliary obstruction or suggest an infiltrative process. Elevated GGT in conjunction with high ALP levels points to hepatobiliary injury. Drug-induced injury may produce a cholestatic pattern but liver ultrasound is usually unremarkable. ${ }^{27}$ If a drug is the suspected cause, the ALP measurement should be repeated after discontinuation of the drug (at 6-8 weeks). If the initial evaluation points to a specific disease, disease-specific markers should be obtained. If the markers are negative and ALP is still elevated, other diagnostic modalities, including liver biopsy, endoscopic retrograde cholangiopancreatography and/or MRCP, should be performed. ${ }^{13}$

Some other diseases that can lead to elevated ALP are primary biliary cholangitis (PBC) and primary sclerosing cholangitis (PSC). PBC is characterized by a persistent immunological attack on the bile ducts that eventually results in cirrhosis and liver failure. The diagnosis of PBC is established when there is no extrahepatic biliary obstruction and no other co-morbid disease affecting the liver present, along with at least two of the following criteria: 1) ALP of at least 1.5 times the ULN; 2) presence of antimitochondrial antibodies (AMA) at a titer of $1: 40$ or higher; and 3 ) histological evidence of the disease process. ${ }^{69}$ PSC is a chronic progressive disease of unknown etiology that is characterized by inflammation, fibrosis, and stricturing of medium and large ducts in the intrahepatic and/or extrahepatic biliary tree that will eventually lead to complications of cholestasis and liver failure. Other than the usual cholestatic pattern of the liver chemistries, the radiographic findings include abnormal-appearing bile ducts with wall thickening, dilations and strictures. Liver biopsy is required for patients with suspected small duct PSC or if other conditions such as an overlap syndrome with $\mathrm{AIH}$ is being considered. ${ }^{70-72}$

\section{Elevated GGT}

GGT, a membrane enzyme, is a marker of hepatobiliary disease. Alcohol and some drugs can induce GGT. Because this enzyme is highly inducible and lacks specificity, extensive evaluation of an isolated elevation in an otherwise asymptomatic patient is not indicated. ${ }^{13}$

\section{Isolated hyperbilirubinemia (elevated bilirubin)}

Unconjugated bilirubin, a product of hemoglobin catabolism, is transported to the liver. In the liver, UDP-glucuronyl transferase conjugates the unconjugated bilirubin with glucuronic acid and conjugated bilirubin is then excreted into the bile. The first step in evaluating a patient with isolated hyperbilirubinemia is to fractionate the bilirubin to determine if the 
level is predominantly conjugated or unconjugated. Unconjugated bilirubin may increase if the production increases (e.g., hemolysis) or hepatic uptake/conjugation decreases (e.g., Gilbert's syndrome).

Increase in conjugated bilirubin is due to decreased excretion into the bile or leakage from the hepatocytes into serum. In the case of unconjugated high bilirubin, the evaluation for hemolysis includes serum hemoglobin and haptoglobin levels and reticulocyte count. When hemolysis is ruled out, other causes of impaired hepatic uptake or conjugation should be considered. These causes include certain drugs (i.e. rifampin), Gilbert's syndrome, and Crigler-Najjar syndromes (type I and II). In the case of conjugated high bilirubin, if the ALP and GGT levels are normal, the two rare inherited conditions of Dubin-Johnson syndrome and Rotor syndrome should be considered. ${ }^{13,27}$

\section{Isolated abnormalities of tests of liver synthetic functions}

Abnormalities in tests such as prothrombin time and serum albumin should be evaluated based on the predominant pattern of liver-associated enzymes abnormalities. Other extrahepatic causes of such abnormalities should be considered as well. ${ }^{11,12}$

\section{When to consult a specialist}

It is reasonable to consult a gastroenterologist/hepatologist if there is an unexplained, persistent liver-associated enzyme elevation of $>2$ times the ULN for ALT/AST or 1.5 times the ULN for ALP. ULN for ALT/AST is considered to be 30 international units/L for men and 20 for women. ${ }^{3}$

\section{Conclusions}

Elevation of liver enzymes is one of the most common problems encountered in the primary care setting and it presents many challenges, even for experienced clinicians. History-taking and physical examination is very important for diagnosis. Laboratory testing can be used based on the pattern of the elevation and the degree of elevation, in order to determine the diagnosis. A systematic approach is recommended to help the clinician find the cause of elevation.

\section{Conflict of interest}

The authors have no conflict of interests related to this publication.

\section{Author contributions}

Conception of article objectives and/or design, drafting of the article, manuscript writing and critical revision (MM, AK), revision of the article for important intellectual content (SKA, SS).

\section{References}

[1] Hultcrantz R, Glaumann H, Lindberg G, Nilsson LH. Liver investigation in 149 asymptomatic patients with moderately elevated activities of serum aminotransferases. Scand J Gastroenterol 1986;21:109-113. doi: 10.3109/ 00365528609034632

[2] Ioannou GN, Boyko EJ, Lee SP. The prevalence and predictors of elevated serum aminotransferase activity in the United States in 1999-2002. Am J Gastroenterol 2006;101:76-82. doi: 10.1111/j.1572-0241.2005.00341.x.
[3] Kwo PY, Cohen SM, Lim JK. ACG clinical guideline: evaluation of abnormal liver chemistries. Am J Gastroenterol 2017;112:18-35. doi: 10.1038/ajg. 2016.517.

[4] Dufour DR, Lott JA, Nolte FS, Gretch DR, Koff RS, Seeff LB. Diagnosis and monitoring of hepatic injury. II. Recommendations for use of laboratory tests in screening, diagnosis, and monitoring. Clin Chem 2000;46:2050-2068.

[5] Dufour DR. Effects of habitual exercise on routine laboratory tests. Clin Chem 1998;44:A136.

[6] Green RM, Flamm S. AGA technical review on the evaluation of liver chemistry tests. Gastroenterology 2002;123:1367-1384. doi: 10.1053/gast.2002. 36061.

[7] Pratt DS, Kaplan MM. Evaluation of abnormal liver-enzyme results in asymptomatic patients. N Engl J Med 2000;342:1266-1271. doi: 10.1056/ NEJM200004273421707.

[8] Gopal DV, Rosen HR. Abnormal findings on liver function tests. Interpreting results to narrow the diagnosis and establish a prognosis. Postgrad Med 2000;107:100-114. doi: 10.3810/pgm.2000.02.869.

[9] Friedman LS, Dienstag JL, Watkins E, Hinkle CA, Spiers JA, Rieder SV, et al. Evaluation of blood donors with elevated serum alanine aminotransferase levels. Ann Intern Med 1987;107:137-144. doi: 10.7326/0003-4819-1072-137.

[10] Hay JE, Czaja AJ, Rakela J, Ludwig J. The nature of unexplained chronic aminotransferase elevations of a mild to moderate degree in asymptomatic patients. Hepatology 1989;9:193-197. doi: 10.1002/hep.1840090205.

[11] Daniel S, Ben-Menachem T, Vasudevan G, Ma CK, Blumenkehl M. Prospective evaluation of unexplained chronic liver transaminase abnormalities in asymptomatic and symptomatic patients. Am J Gastroenterol 1999;94: 3010-3014. doi: 10.1111/j.1572-0241.1999.01451.x.

[12] Harrison SA, Kadakia S, Lang KA, Schenker S. Nonalcoholic steatohepatitis: what we know in the new millennium. Am J Gastroenterol 2002;97: 2714-2724. doi: 10.1111/j.1572-0241.2002.07069.x.

[13] Aragon G, Younossi ZM. When and how to evaluate mildly elevated liver enzymes in apparently healthy patients. Cleve Clin J Med 2010;77: 195-204. doi: 10.3949/ccjm.77a.09064.

[14] Rude MK, Kerr T, Lisker-Melman M. Liver diseases. In: Godara H, Hirbe A, Nassif $M$, Otepka $H$, Rosenstock $A$, ed. The washington manual of medical therapeutics ( $34^{\text {th }}$ Edition). Washington: Lippincott Williams \& Wilkins, 2014:664-721.

[15] Verslype C. Evaluation of abnormal liver-enzyme results in asymptomatic patients. Acta Clin Belg 2004;59:285-289. doi: 10.1179/acb.2004.042.

[16] Das A, Post AB. Should liver biopsy be done in asymptomatic patients with chronically elevated transaminases: A costutility analysis. Gastroenterology 1998;114:A9. doi: 10.1016/S0016-5085(98)80035-8.

[17] Sorbi D, McGill DB, Thistle JL, Therneau TM, Henry J, Lindor KD. An assessment of the role of liver biopsies in asymptomatic patients with chronic liver test abnormalities. Am J Gastroenterol 2000;95:3206-3210. doi: 10.1111/ j.1572-0241.2000.03293.x.

[18] Cohen JA, Kaplan MM. The SGOT/SGPT ratio-an indicator of alcoholic liver disease. Dig Dis Sci 1979;24:835-838. doi: 10.1007/BF01324898.

[19] Diehl AM, Potter J, Boitnott J, Van Duyn MA, Herlong HF, Mezey E. Relationship between pyridoxal 5 '-phosphate deficiency and aminotransferase levels in alcoholic hepatitis. Gastroenterology 1984;86:632-636.

[20] Ruhl CE, Everhart JE. Joint effects of body weight and alcohol on elevated serum alanine aminotransferase in the United States population. Clin Gastroenterol Hepatol 2005;3:1260-1268. doi: 10.1016/S1542-3565(05)00743-3.

[21] Alter HJ. To C or not to C: these are the questions. Blood 1995;85: 1681-1695.

[22] Everhart JE. Digestive diseases in the United States: Epidemiology and impact. Maryland: NIH Publication, 1994:94-1447.

[23] Dancygier H, Rogart JN. Approach to the patient with abnormal liver enzymes. In: Dancygier H, ed. Clinical hepatology. Verlag Berlin Heidelberg: Springer, 2010:533-547. doi: 10.1007/978-3-540-93842-2_49.

[24] Raimondo G, Navarra G, Mondello S, Costantino L, Colloredo G, Cucinotta E, et al. Occult hepatitis $B$ virus in liver tissue of individuals without hepatic disease. J Hepatol 2008;48:743-746. doi: 10.1016/j.jhep.2008.01.023.

[25] Schiff ER, de Medina M, Kahn RS. New perspectives in the diagnosis of hepatitis C. Semin Liver Dis 1999;19 Suppl 1:3-15.

[26] Halfon $P$, Bourlière $M$, Pénaranda $G$, Khiri $H$, Ouzan D. Real-time PCR assays for hepatitis $\mathrm{C}$ virus (HCV) RNA quantitation are adequate for clinical management of patients with chronic HCV infection. J Clin Microbiol 2006;44: 2507-2511. doi: 10.1128/JCM.00163-06.

[27] Giannini EG, Testa R, Savarino V. Liver enzyme alteration: a guide for clinicians. CMAJ 2005;172:367-379. doi: 10.1503/cmaj.1040752.

[28] Williams AL, Hoofnagle JH. Ratio of serum aspartate to alanine aminotransferase in chronic hepatitis. Relationship to cirrhosis. Gastroenterology 1988; 95:734-739. doi: 10.1016/S0016-5085(88)80022-2.

[29] Giannini E, Botta F, Fasoli A, Ceppa P, Risso D, Lantieri PB, et al. Progressive liver functional impairment is associated with an increase in AST/ALT ratio. Dig Dis Sci 1999;44:1249-1253. doi: 10.1023/A:1026609231094. 
[30] Giannini E, Risso D, Botta F, Chiarbonello B, Fasoli A, Malfatti F, et al. Validity and clinical utility of the aspartate aminotransferase-alanine aminotransferase ratio in assessing disease severity and prognosis in patients with hepatitis C virus-related chronic liver disease. Arch Intern Med 2003;163: 218-224. doi: 10.1001/archinte.163.2.218.

[31] Giannini E, Botta F, Testa E, Romagnoli P, Polegato S, Malfatti F, et al. The 1 -year and 3-month prognostic utility of the AST/ALT ratio and model for end-stage liver disease score in patients with viral liver cirrhosis. Am J Gastroenterol 2002;97:2855-2860. doi: 10.1111/j.1572-0241.2002.07053.x.

[32] Sorbi D, Boynton J, Lindor KD. The ratio of aspartate aminotransferase to alanine aminotransferase: potential value in differentiating nonalcoholic steatohepatitis from alcoholic liver disease. Am J Gastroenterol 1999;94: 1018-1022. doi: 10.1111/j.1572-0241.1999.01006.x.

[33] Bacon BR, Farahvash MJ, Janney CG, Neuschwander-Tetri BA. Nonalcoholic steatohepatitis: an expanded clinical entity. Gastroenterology 1994;107: 1103-1109. doi: 10.1016/0016-5085(94)90235-6.

[34] Brunt EM. Nonalcoholic steatohepatitis. Semin Liver Dis 2004;24:3-20. doi: 10.1055/s-2004-823098.

[35] Diehl AM, Goodman Z, Ishak KG. Alcohollike liver disease in nonalcoholics. A clinical and histologic comparison with alcohol-induced liver injury. Gastroenterology 1988;95:1056-1062. doi: 10.1016/0016-5085(88)90183-7.

[36] Angulo P, Keach JC, Batts KP, Lindor KD. Independent predictors of liver fibrosis in patients with nonalcoholic steatohepatitis. Hepatology 1999;30: 1356-1362. doi: 10.1002/hep.510300604.

[37] Mofrad P, Contos MJ, Haque M, Sargeant C, Fisher RA, Luketic VA, et al. Clinical and histologic spectrum of nonalcoholic fatty liver disease associated with normal ALT values. Hepatology 2003;37:1286-1292. doi: 10.1053/ jhep.2003.50229.

[38] Matteoni CA, Younossi ZM, Gramlich T, Boparai N, Liu YC, McCullough AJ. Nonalcoholic fatty liver disease: a spectrum of clinical and pathological severity. Gastroenterology 1999;116:1413-1419. doi: 10.1016/S00165085(99)70506-8.

[39] Eriksson S, Eriksson KF, Bondesson L. Nonalcoholic steatohepatitis in obesity: a reversible condition. Acta Med Scand 1986;220:83-88. doi: 10. 1111/j.0954-6820.1986.tb02733.x.

[40] Lavine JE. Treatment of obesity-induced steatohepatitis with vitamin E. Gastroenterology 1998;114:A1284-A1285. doi: 10.1016/S0016-5085(98) 85214-1.

[41] Powell LW, George DK, MCDonnell SM, Kowdley KV. Diagnosis of hemochromatosis. Ann Intern Med 1998;129:925-931. doi: 10.7326/0003-4819129-11_Part_2-199812011-00002.

[42] Tavill AS; American Association for the Study of Liver Diseases; American College of Gastroenterology; American Gastroenterological Association. Diagnosis and management of hemochromatosis. Hepatology 2001;33: 1321-1328. doi: 10.1053/jhep.2001.24783.

[43] Guyader D, Jacquelinet C, Moirand R, Turlin B, Mendler MH, Chaperon J, et al. Noninvasive prediction of fibrosis in $\mathrm{C} 282 \mathrm{Y}$ homozygous hemochromatosis. Gastroenterology 1998;115:929-936. doi: 10.1016/S0016-5085(98)70265-3.

[44] Morrison ED, Kowdley KV. Genetic liver disease in adults. Early recognition of the three most common causes. Postgrad Med 2000;107:147-159. doi: 10. 3810/pgm.2000.02.872.

[45] Krawitt EL. Autoimmune hepatitis. N Engl J Med 1996;334:897-903. doi: 10. 1056/NEJM199604043341406.

[46] Manns MP, Bahr MJ. Recurrent autoimmune hepatitis after liver transplantationwhen non-self becomes self. Hepatology 2000;32:868-870. doi: 10.1053/jhep. 2000.19149.

[47] Czaja AJ. Natural history, clinical features, and treatment of autoimmune hepatitis. Semin Liver Dis 1984;4:1-12. doi: 10.1055/s-2008-1040641.

[48] Alvarez F, Berg PA, Bianchi FB, Bianchi L, Burroughs AK, Cancado EL, et al. International Autoimmune Hepatitis Group Report: review of criteria for diagnosis of autoimmune hepatitis. J Hepatol 1999;31:929-938. doi: 10. 1016/S0168-8278(99)80297-9.

[49] Limdi JK, Hyde GM. Evaluation of abnormal liver function tests. Postgrad Med ] 2003;79:307-312. doi: 10.1136/pmj.79.932.307.
[50] Bardella MT, Vecchi M, Conte D, Del Ninno E, Fraquelli M, Pacchetti S, et al. Chronic unexplained hypertransaminasemia may be caused by occult celiac disease. Hepatology 1999;29:654-657. doi: 10.1002/hep.510290318.

[51] Lo Iacono O, Petta S, Venezia G, Di Marco V, Tarantino G, Barbaria F, et al. Anti-tissue transglutaminase antibodies in patients with abnormal liver tests: is it always coeliac disease? Am J Gastroenterol 2005;100:2472-2477. doi: 10.1111/j.1572-0241.2005.00244.x.

[52] Volta U, De Franceschi L, Lari F, Molinaro N, Zoli M, Bianchi FB. Coeliac disease hidden by cryptogenic hypertransaminasaemia. Lancet 1998;352: 26-29. doi: 10.1016/S0140-6736(97)11222-3.

[53] Farrell RJ, Kelly CP. Diagnosis of celiac sprue. Am J Gastroenterol 2001;96: 3237-3246. doi: 10.1111/j.1572-0241.2001.05320.x.

[54] Lee WM. Drug-induced hepatotoxicity. N Engl J Med 2003;349:474-485. doi: 10.1056/NEJMra021844.

[55] Fogden E, Neuberger J. Alternative medicines and the liver. Liver Int 2003; 23:213-220. doi: 10.1034/j.1600-0676.2003.00843.x.

[56] Shad JA, Chinn CG, Brann OS. Acute hepatitis after ingestion of herbs. South Med J 1999;92:1095-1097. doi: 10.1097/00007611-199911000-00011.

[57] Kessler WR, Cummings OW, Eckert G, Chalasani N, Lumeng L, Kwo PY. Fulminant hepatic failure as the initial presentation of acute autoimmune hepatitis. Clin Gastroenterol Hepatol 2004;2:625-631. doi: 10.1016/ S1542-3565(04)00246-0.

[58] Whitehead MW, Hawkes ND, Hainsworth I, Kingham JG. A prospective study of the causes of notably raised aspartate aminotransferase of liver origin. Gut 1999;45:129-133. doi: 10.1136/gut.45.1.129.

[59] Seeto RK, Fenn B, Rockey DC. Ischemic hepatitis: clinical presentation and pathogenesis. Am J Med 2000;109:109-113. doi: 10.1016/S0002-9343 (00)00461-7.

[60] Fuchs S, Bogomolski-Yahalom V, Paltiel O, Ackerman Z. Ischemic hepatitis: clinical and laboratory observations of 34 patients. J Clin Gastroenterol 1998;26:183-186. doi: 10.1097/00004836-199804000-00007.

[61] Singer AJ, Carracio TR, Mofenson HC. The temporal profile of increased transaminase levels in patients with acetaminophen-induced liver dysfunction. Ann Emerg Med 1995;26:49-53. doi: 10.1016/S0196-0644(95)70237-7.

[62] Sellers EM, Freedman F. Treatment of acetaminophen poisoning. Can Med Assoc J 1981;125:827-829.

[63] Marcellin P. Hepatitis C: the clinical spectrum of the disease. J Hepatol 1999; 31 Suppl 1:9-16. doi: 10.1016/S0168-8278(99)80368-7.

[64] Wedemeyer H, Jäckel E, Wiegand J, Cornberg M, Manns MP. Whom? When? How? Another piece of evidence for early treatment of acute hepatitis C. Hepatology 2004;39:1201-1203. doi: 10.1002/hep.20221.

[65] Roberts EA, Schilsky ML; Division of Gastroenterology and Nutrition, Hospital for Sick Children, Toronto, Ontario, Canada. A practice guideline on Wilson disease. Hepatology 2003;37:1475-1492. doi: 10.1053/jhep.2003.50252.

[66] Fortson WC, Tedesco FJ, Starnes EC, Shaw CT. Marked elevation of serum transaminase activity associated with extrahepatic biliary tract disease. J Clin Gastroenterol 1985;7:502-505. doi: 10.1097/00004836-19851200000012.

[67] Anciaux ML, Pelletier G, Attali P, Meduri B, Liguory C, Etienne JP. Prospective study of clinical and biochemical features of symptomatic choledocholithiasis. Dig Dis Sci 1986;31:449-453. doi: 10.1007/BF01320306.

[68] Ferenci P, Caca K, Loudianos G, Mieli-Vergani G, Tanner S, Sternlieb I, et al. Diagnosis and phenotypic classification of Wilson disease. Liver Int 2003;23: 139-142. doi: 10.1034/j.1600-0676.2003.00824.x.

[69] Lindor KD, Gershwin ME, Poupon R, Kaplan M, Bergasa NV, Heathcote EJ, et al. Primary biliary cirrhosis. Hepatology 2009;50:291-308. doi: 10 . 1002/hep.22906.

[70] Lee YM, Kaplan MM. Primary sclerosing cholangitis. N Engl ] Med 1995;332: 924-933. doi: 10.1056/NEJM199504063321406.

[71] Angulo P, Lindor KD. Primary sclerosing cholangitis. Hepatology 1999;30: 325-332. doi: 10.1002/hep.510300101.

[72] Chapman R, Fevery J, Kalloo A, Nagorney DM, Boberg KM, Shneider B, et al. Diagnosis and management of primary sclerosing cholangitis. Hepatology 2010;51:660-678. doi: 10.1002/hep.23294. 